\title{
LA INFLUENCIA DE LA WEB EN LA REPUTACIÓN ONLINE: EL CASO DE TRIPADVISOR Y MINUBE
}

\author{
ANA BELÉN BASTIDAS MANZANO ${ }^{1}$ \\ Departamento de Turismo y Marketing \\ Facultad de Ciencias Económicas y Empresariales \\ Universidad a Distancia de Madrid \\ JUAN SÁNCHEZ FERNÁNDEZ ${ }^{2}$ \\ Departamento de Investigación y Comercialización de mercados \\ Facultad de Ciencias Económicas y Empresariales \\ Universidad de Granada \\ LUIS ALBERTO CASADO ARANDA \\ Departamento de Turismo y Marketing \\ Facultad de Ciencias Económicas y Empresariales \\ Universidad a Distancia de Madrid
}

Resumen:

El turismo en España representa el principal sector económico del país y se posiciona como un gran impulsor de arrastre de nuestra economía. El auge de las nuevas tecnologías y el proceso de digitalización (conocido como Web 2.0) está teniendo importantes repercusiones en la forma con que las organizaciones turísticas ofrecen sus servicios y los clientes interactúan con ellas. El presente trabajo pretende explorar la importancia de la Web en la generación de una reputación online en las empresas del sector turístico. Concretamente, compara la influencia de dos plataformas de comunidades virtuales de viajeros (Tripadvisor y MiNube) como generadoras de contenido de recomendaciones en la creación de la imagen y reputación de un destino turístico. El usuario como generador de contenido se convierte entonces en una pieza fundamental para dar valor a las empresas del sector. Es aquí donde la sociología del turismo ocupa un papel fundamental en el estudio del nuevo turista en el entorno de la Travel 2.0. Los resultados del estudio corroboran la importancia del contenido web generado por usuarios como factores clave en la reputación del destino turístico y relevancia en la gestión de los viajes.

Palabras clave: Web 2.0, reputación online, boca-oído electrónico, turismo 2.0, comunidades virtuales.

anabelen.bastidas@udima.es

sanchezf@ugr.es

luisalberto.casado@udima.es 


\title{
THE INFLUENCE OF WEBSITES ON ONLINE REPUTATION: THE CASE OF TRIPADVISOR AND MINUBE
}

\begin{abstract}
:
Tourism constitutes the most influential economic sector in Spain and it is posiTioned as a main driver of the Spanish economy. The recent advance of new technologies and the digitization process (known as Web 2.0) are strongly affecting the way in which touristic firms offer their services and that in which customers interact with them. The current research precisely aims to explore the importance of the Web in generating online reputation between firms belonging to the touristic sector. Specifically, it compares the influence of two platforms of virtual communities of travelers (namely, Tripadvisor and MiNube) as generators of content recommendations in the creation of the image and reputation of a tourist destination. Within such context, the user becomes in a key piece in providing value to tourist firms. At such context, Sociology of Tourism represents a key factor when studying the new tourist within the Web 2.0. The findings of our study corroborate the importance of the web content triggered by users as a key variable influencing the reputation of the tourist destination as well as the relevance in the travel management.
\end{abstract}

Key words: Web 2.0, online reputation, E-WOM, tourism 2.0, virtual communities

\section{INTRODUCCIÓN: LA IMPORTANCIA DE LA WEB COMO CANAL DE COMUNICACIÓN PARA LA EMPRESA TURÍSTICA}

Según los últimos datos del Instituto de Estudios Turísticos ${ }^{4}$ (2018), la balanza de pagos de España para el año 2017 destaca por el superávit turístico. A su vez, los datos del empleo en el sector servicios mantienen su tendencia al crecimiento, en torno al $4 \%$ en los últimos trimestres. De hecho, se confirma la tendencia a una progresiva estabilización en el comportamiento del conjunto del sector turístico español: la actividad turística ha vuelto a crecer en los tres meses de verano, si bien a un ritmo del $+1,1 \%$, por debajo del crecimiento de la economía española $(+2,6 \%)$, acorde a la desaceleración en el crecimiento de los ingresos turísticos extranjeros y el menor empuje del esperado de la demanda nacional (Exceltur , 2018). Estos datos revelan la importancia del turismo en la economía y empleo de España.

Por su parte, las nuevas tecnologías de la información y las comunicaciones (TIC) han transformado el mercado de las comunicaciones y en especial el sector turístico. En los últimos años, Internet se ha convertido en el canal principal para la búsqueda y propagación de información (Standing, tang-taye y Boyer 2014). El turismo no ha sido una excepción e Internet se ha posicionado como la mayor fuente de información para los viajeros (Pan, MacLaurin y Crotts 2007). En este contexto, Internet se ha convertido en el principal

\footnotetext{
5 https://bit.ly/2LgJx7I (Acceso 05/12/2018)

https://www.exceltur.org/\# (Acceso 05/12/2018)
} 
suministrador de información y publicidad, siendo el medio que usan los turistas por excelencia para la elección de su destino vacacional.

El desarrollo de dispositivos móviles que permiten el uso de la Red fuera del domicilio facilita la constante búsqueda de comunicación del usuario con otros usuarios o con los administradores turísticos. Teléfonos móviles con conexión a Internet (smartphone), tabletas y ordenadores portátiles facilitan el desarrollo tecnológico de un sector como el turismo que está a la vanguardia en el campo de la innovación.

El hecho en sí de buscar información sobre la reserva, el transporte, el alojamiento y el destino es un proceso donde tradicionalmente proveedores, intermediarios y consumidores tenían su propio papel utilizando sus propios sistemas de información específicos (Henriksson, 2005). Sin embargo, la estructura de la industria del turismo está cambiando (Ricci, Rokach y Shapira 2015), y esto puede ser fácil de comprender si nos centramos en el factor de distribución: el comercio electrónico es muy apropiado para la industria del turismo ya que los consumidores no están por lo general cerca de los lugares donde se produce esencialmente la producción. De hecho, las TIC han contribuido al crecimiento masivo del turismo y el aumento del volumen de la oferta y la demanda pero, ante todo, han ido cambiando la forma en que las empresas turísticas llevan a cabo sus negocios. Internet ha cambiado el comportamiento de los consumidores a la hora de comprar un viaje y la Web 2.0 facilita el intercambio de información entre turistas.

Así pues, la tecnología provee de información al cliente en la gestión del proceso de viaje:

1) En el pre-viaje al tomar una decisión, los turistas usan la Web para buscar información y confirmar con familiares o amigos sus posibles destinos, opciones de transporte y alojamiento que ellos consideran como una parte importante de su viaje. En esta fase el uso de redes sociales online y de páginas Webs especializadas será básico para la correcta elección del destino.

2) Durante el desarrollo del viaje, los turistas usan las redes sociales y otros recursos para guardar y compartir información.

3) En la última fase de su experiencia de viaje, conocida como la etapa de recolección, los turistas usan la Web para poner sus historias de viajes y fotografías, recordar momentos especiales y entablan una comunicación boca-oído (Gretzel 2006).

En este trabajo profundizamos en la idea propuesta por Arsal et al. (2010), quienes sugirieron que las comunidades de viajes online influyen en la decisión del proceso de compra, publicando problemas o sugerencias ocurridos durante el viaje, influyendo con sus opiniones (positivas o negativas) en la reputación online de destinos y empresas turísticas. El último estudio anual de redes sociales realizado por IAB Spain (2018) determina que un $85 \%$ de los internautas de 16-65 años utilizan redes sociales, lo que representa más de 25.5 millones de usuarios en nuestro país. Según el Estudio Social Travel España (2013) desarrollado por Territorio Creativo y NH Hoteles, casi un 60\% de los españoles consulta Web de viajes como Tripadvisor o MiNube para seleccionar un destino turístico. Un $66 \%$ de los españoles consulta sitios web oficiales de destinos para corroborar datos e informarse de

$\begin{array}{ll}6 & \text { https://iabspain.es/estudio/estudio-anual-de-ecommerce-2018-version-completa/ (Acceso 10/12/2018) } \\ \text { https://www.concepto05.com/2013/05/i-estudio-social-travel-espana/ (Acceso 11/12/2018) }\end{array}$ 
actividades, alojamientos y datos prácticas del lugar de destino que les facilitarán el proceso del viaje.

Así, los activos digitales de destinos turísticos mantienen una posición preeminente en varias de las fases de la gestión de un viaje, en particular en la primera fase, la de planificación. Según datos del último estudio de Minerva Travel 2017 (realizado por Google Spain), el 67\% de los viajeros se inspiraron en las opiniones y comentarios online para hacer la reserva de sus viajes. Estos datos corroboran, por un lado, la importancia del uso de las tecnologías online (Internet y redes sociales) para la reserva a inspiración de sus viajes, a su vez que, ponen de manifiesto que la tendencia de estos datos es ir aumentando el porcentaje de usuarios que usa la Red para el proceso de compra en el sector servicios.

En el trabajo de Hernández-Méndez, Muñoz-Leiva y Sánchez-Fernández (2015) se hace referencia a dos comunidades virtuales como TripAdvisor y Minube, usadas cada día más por el consumidor para la elección de sus viajes. Dada la importancia reciente de dichas comunidades virtuales en la actualidad en España (Lee y Hyun 2018; Ruiz-Mafe et al. 2018), hemos considerado de interés profundizar en ellas.

Siguiendo esta línea, uno de los objetivos del trabajo es comparar el contenido, información y servicios ofrecidos por dos comunidades virtuales de viajeros, por un lado, el caso de Tripadvisor, la Web de viajes más importante a nivel mundial y, por otro lado, el caso de MiNube, una empresa de carácter español con cierta similitud a Tripadvisor que está revolucionando el mundo de los viajes. Ambas Webs influyen directamente en la elección de un destino por parte de un cliente, decidiendo su compra en función de los comentarios vistos en la página. Mediante dicha comparación, este trabajo pretende corroborar el papel de la Web como gestora de la reputación online en las empresas turísticas. En este caso, la reputación de una empresa en los medios online será un valor a tener en cuenta, puesto que cada día son más los clientes que deciden una empresa turística u otra en función de la puntuación de las mismas en las principales páginas Webs de viajes.

El papel del marketing online en un entorno 2.0 para un sector tan cambiante y dependiente del entorno, será fundamental para la optimización de los beneficios empresariales. Conocer la importancia de la reputación en la red posibilitará al sector empresarial la mejora en los procesos de comunicación y adaptación a las necesidades tanto presentes como futuras del consumidor.

\section{MARCO TEÓRICO Y CONCEPTUAL}

\subsection{La Web 2.0.}

Comprender el desarrollo online del turismo es una de las premisas básicas del trabajo que se plantea, para lo cual, necesitamos entender qué es la Web 2.0 y la importancia que tiene para el sector servicios. Este término describe a una Web la cual está cada vez más influenciada por la Web inteligente, que permite a los usuarios contribuir al desarrollo, calificación, colaboración y distribución del contenido así como, la personalización de las aplicaciones de Internet (Vickery y Wunsch-Vincent 2007).

Las herramientas de la Web 2.0 son muy usadas por los turistas para obtener información sobre destinos turísticos y compartir sus experiencias. El contenido digital creado

https://bit.ly/2DZvC2n (Acceso 12/12/2018) 
por los turistas ha incrementado el conocimiento sobre el destino y la imagen creada sobre éste (Tussyadiah y Fesenmaier 2009).

Las herramientas de la Web 2.0 son muy usadas por los turistas para obtener información sobre destinos turísticos y compartir sus experiencias. El contenido digital creado por los turistas ha incrementado el conocimiento sobre el destino y la imagen creada sobre éste (Tussyadiah y Fesenmaier 2009). Para Gomis (2008) la Travel 2.0 "Se trata de un nuevo modelo de Internet donde consultar, publicar, discutir, compartir y distribuir información sobre turismo cuyo contenido sea creado por los usuarios interactuando entre ellos". Hablamos de una Web creada por usuarios para usuarios, ya no es el destino y/o empresa la que se dedica a enumerar las bondades de su negocio, sino, que es el propio turista el que tras su experiencia le cuenta al resto de la comunidad virtual qué hacer, dónde comer o qué ver en un destino.

La evolución de la llamada Web 2.0 y su relación con el turismo se debe a los siguientes factores:

- un potente y asequible hardware y software

- una red más rápida

- la facilidad de utilizar herramientas para crear y compartir contenidos

- una mayor alfabetización digital de los consumidores

- el aumento de plataformas portátiles e inalámbricas (Parameswaran y Whinston 2007)

El desarrollo de la Web 2.0 ha propiciado el auge de opiniones sobre empresas y destinos turísticos en Internet. Las redes sociales, las comunidades virtuales de viajeros, los blogs, los canales de vídeo, etc. han cambiado la forma en que los usuarios preparan y compran sus vacaciones. Ya no hace falta acudir a una agencia de viajes tradicional o ser un experto en tecnología para hacer una reserva de transporte o alojamiento. El uso de la Web por parte del turista dependerá de la capacidad que tenga dicha Web para satisfacer las necesidades del usuario (turista) (Luque-Martínez et al. 2007)

Burgess, Parish y Alcock (2011) afirmaron que las personas ponen más confianza en los comentarios de las agencias de viajes y viajeros en sitios de terceros dedicados a viajar. En un estudio sobre la credibilidad de comentarios en línea, Kusumasondjaja, Shanka y Marchegiani (2012) encontraron que cuando se muestra la identidad de los revisores, los comentarios negativos se consideran creíbles y las críticas positivas se consideran fiables.

Es en este contexto donde el papel de las redes sociales y las comunidades virtuales aportan un papel fundamental, al contribuir a la Web con opiniones de usuarios y ofrecer información actualizada y de primera persona sobre los servicios turísticos.

\subsection{Redes sociales y Comunidades Virtuales de Viajeros}

El panorama de la comunicación ha sido objeto de una inmensa transformación en la última década (Mangold y Faulds 2009). Los medios de comunicación social, es decir, las redes sociales, reemplazan cada vez más a los medios de comunicación tradicionales, y el crecimiento acerca de esta nueva comercialización parece ilimitado 
No existe una definición universalmente adoptada de Social Media, por su parte, Solis y Breakenridge (2009), señalan que podemos definir las redes sociales como "cualquier herramienta o servicio que utiliza Internet para facilitar conversaciones". El auge del Social Media tiene implicaciones particularmente significativas en la industria de los viajes y turismo. Como una de las funciones principales, las redes sociales permiten el intercambio de información entre personas de diferentes partes del mundo. Las redes sociales están trayendo cambios a los patrones de comunicación y las relaciones interpersonales (Jansson 2018; Moro y Rita 2018).

Esta tendencia de los consumidores a convertirse en fans de las marcas en las redes sociales y el uso de los medios sociales como una fuente cada vez mayor de información sobre marcas conduce a la suposición de que los medios de comunicación social, además de los instrumentos de comercialización tradicional, ejercen un impacto importante en el éxito y reputación de una marca. Este crecimiento de los medios sociales parece ilimitado por ahora (Trusov, Bodapati y Bucklin 2010).

Desde que los medios de comunicación social ofrecen una oportunidad para los consumidores de hablar con cientos o incluso miles de otros consumidores de todo el mundo, las empresas ya no son la única fuente de comunicación de la marca. Además, los consumidores se están alejando de medios tradicionales como la televisión, la radio o la prensa y utilizan cada vez más las redes sociales para dar a conocer sus productos/servicios (Mangold y Faulds 2009).

Por su parte, las comunidades virtuales han surgido como desarrollo de las redes sociales online y su facilitad para entablar conversación online. El mundo del turismo ha sufrido un gran desarrollo, siendo muchas las personas que hoy en día en la red se dedican de manera casi profesional a la creación de blog de viajes donde asesorar a futuros viajeros hacía cualquier lugar del mundo.

Tanto el uso de la tecnología de Internet como las comunidades de viajes online ofrecen una oportunidad a los turistas para buscar información de viajes, establecer relaciones con otros, y de esta forma, tomar la decisión sobre viaje, conociendo siempre los precios en los distintos puntos de venta (Arsal et al. 2010).

Por su parte, las comunidades virtuales representan un nuevo tipo de formación social en Internet. Propician la apertura del poder de la tecnología para conectar a las personas, una oportunidad sin precedente para la interacción social y el desarrollo de las relaciones entre las personas con intereses comunes, con independencia del lugar geográfico y del tiempo (Armstrong y Hagel 2000). Las comunidades virtuales han actuado tradicionalmente como un lugar de encuentro virtual entre personas para compartir intereses, construir relaciones o establecer transacciones de servicios turísticos. Esta conducta es fruto de una industria intensiva en información, los consumidores pueden utilizar Internet para acceder a la información de forma simple, de manera eficiente, y recibir tarifas más flexibles, ahorrar tiempo y reducir las negociaciones en procesos tales como reserva de entradas y alojamiento. Estas características han contribuido a la popularidad de los portales de viajes online.

Los productos en los que interviene el cliente son muchos más exitosos tras la participación de éste. Esto es particularmente cierto en la industria turística ya que los clientes están profundamente involucrados en la producción de un servicio/destino turístico con el fin de aumentar su experiencia (Consoli y Baglieri 2009). Tal y como se ha venido considerando, el comportamiento del sector turístico difiere del resto de productos tangibles, puesto que en este caso el factor experiencia va a ser un elemento fundamental. La participación del cliente 
en el sector servicios es especialmente útil para obtener información sobre sus necesidades y deseos con el fin de desarrollar un producto/servicio turístico diferenciador. El desarrollo de Internet ha facilitado el espacio donde reunir y compartir esa información. Los clientes pueden interactuar con empresas y con otros usuarios y pueden conseguir más información que les permite reducir la asimetría de información y tener más poder que tenían en la era pretecnológica. Así pues, recientes investigaciones (Bechwati y Nasr 2011; Burgess, Parish y Alcock 2011; Di Pietro y Pantano 2012; Benítez, López y Gutiérrez 2018) indican que los comentarios on line influyen en las decisiones de compra de los individuos.

Por lo tanto, las comunidades virtuales permiten a las personas interactuar para satisfacer sus propias necesidades. Las empresas pueden aprovechar estas comunidades al proporcionar un marco adecuado donde los clientes pueden participar en charlas sociales, debates o foros. Una comunidad virtual turística debe ser una integración de contenidos y comunicación directa, con una tecnología fácil y una diversidad de información. En consecuencia, el diseño adecuado de la comunidad turística debe basarse en una comprensión completa de funcionalidad de los consumidores sociales y sus necesidades psicológicas y cómo estas interactúan entre sí.

La socialización es un elemento importante en el proceso de creación del servicio (Bitner, Booms y Tetreault 1990), en el que por supuesto juegan un papel principal las comunidades virtuales. Por lo tanto, se entiende como los empleados del sector servicios influyen en las percepciones del turista (van Dolen et al. 2002). La base de las comunidades virtuales es que se desarrollan por y para personas, por lo tanto, el componente social será un factor clave en el desarrollo de la comunicación. De ahí los conceptos de boca-oído y reputación, tan importantes para el sector turístico en su proceso de comercialización online.

\subsection{El Boca- Oído (WOM) y el Boca- Oído electrónico (E-WOM)}

Dada la naturaleza intangible del turismo, es habitual que un viajero potencial que nunca ha estado en un destino con anterioridad, capte la información suministrada por otras personas a través de redes sociales en Internet (Saranow, 2004). Un número significativo de sitios Web en los que los usuarios generan el contenido relacionados con los viajes están recibiendo el reconocimiento de los usuarios, puesto que estos abandonan las fuentes más tradicionales de información (por ejemplo, la publicidad impulsada por las empresas) a favor del "boca a boca" (WOM por sus iniciales en inglés) (Bray, 2007).

Westbrook (1987) define el WOM como la comunicación informal entre los consumidores sobre las características, propiedad y uso de un producto o servicio, en el que las fuentes se consideran independientes de la influencia comercial. No obstante, el desarrollo de Internet ha dado lugar a otro término, el e-WOM. Hennig-Thurau et al. (2006) o definieron como cualquier declaración positiva o negativa creada por clientes potenciales, actuales o antiguos acerca de un producto, servicio o empresa, y que se ofrece a una multitud de personas e instituciones a través de Internet. Los consumidores de viajes buscan otras opiniones para gestionar el riesgo percibido de un producto intangible. La literatura previa apoya la influencia del boca a boca y la recomendación de los clientes en el establecimiento de comercio electrónico (Smith, Menon y Sivakumar 2005). En este contexto, los turistas se convierten en co-vendedores, co-diseñadores, co-productores y compañeros de los consumidores de viajes y experiencias de turismo.

Así pues, el WOM ha resultado ser uno de las más importantes fuentes de información a la hora de planear un viaje, necesitando por parte del consumidor de la Web ver 
principalmente independencia de la fuente que escribe el mensaje. Este WOM da lugar a la llamada reputación (tanto off como online) de la empresa.

\subsection{Reputación}

Yacouel y Fleischer (2012) mostraron que los intermediarios online, especialmente agencias de viajes online, y la información de los clientes en sus sitios web pueden afectar a la reputación del hotel. Sin embargo, los comentarios falsos podrían afectar a los resultados de marketing en función del efecto en la percepción del cliente de la credibilidad, la fiabilidad y la confianza. Yoo y Gretzel (2009) establecen que las diferencias identificadas en el léxico, en particular el uso de la primera persona, la mención de los nombres de marca y los sentimientos son factores útiles para identificar falsas opiniones. O'Connor (2010) proporcionó información acerca de la poca evidencia de falsas opiniones de hoteles en varios sitios web de opiniones, incluso con voto negativo, y recomendó que los hoteles debieran participar activamente en el tratamiento de los comentarios negativos. Los comentarios negativos son una oportunidad para interactuar con el cliente e intentar solucionar posibles malentendidos. El resto de potenciales consumidores podrá de esta manera llevarse una completa opinión del problema en función de la solución establecida.

De la misma forma que las personas, las empresas poseen reputación, definida como un conjunto de atributos económicos y no económicos unidos a la organización y generados a partir de sus acciones pasadas (Weigelt y Camerer 1988). La reputación de la organización se configuraría a partir de los juicios de sus diferentes públicos, quienes analizan las diferentes señales emitidas por la empresa, tanto de forma controlada como de manera involuntaria. Dichas señales, según (Fombrun 2017) podrían resumirse en señales de mercado (cuota de mercado, etc); señales contables (rentabilidad, riesgo financiero, etc); señales institucionales (propiedad institucional de la empresa, responsabilidad social, visibilidad en los medios de comunicación, etc.) y, finalmente, señales estratégicas (diferenciación y diversificación). Así, "la reputación vendría a ser un agregado compuesto a partir de las operaciones llevadas a cabo por la entidad a lo largo de su vida, una noción histórica, y requiere consistencia de las acciones de la organización a lo largo de un espacio de tiempo prolongado para su formación" (Herbig, Milewicz y Golden 1994).

Hablar de la reputación online (e-reputation) es hablar de ese elemento de la reputación que deriva específicamente de los contactos online (Chun y Davies 2001). Algunos autores como Jarvenpaa, Tractinsky y Saarinen (1999) consideran más importante la reputación en la red que aquella que se genera en contextos off-line, puesto que, un mal comentario en la Red puede llegar a miles de personas. Los consumidores de viajes buscan otras opiniones para gestionar el riesgo percibido de un producto intangible. La literatura previa apoya la influencia del boca a boca y la recomendación de los clientes en el establecimiento de comercio electrónico (Smith, Menon y Sivakumar 2005).

El rendimiento del sistema de reputación se basa en dos conceptos principales: reputación y confianza (Jøsang, Ismail y Boyd 2007). En los entornos virtuales que se aplican los sistemas de reputación, los usuarios pueden decidir si confían en un vendedor online desde el momento de establecer su reputación. Los sistemas de reputación ofrecen una opción para ayudar a los usuarios a identificar relaciones de confianza en Internet, lo que les permite evaluar la reputación del vendedor en línea.

Por lo tanto, la reputación es el resultado de un conjunto de juicios públicos que mejoran (o empeoran) con el tiempo, y es compartida en términos sociales por parte de los diversos grupos de interés (clientes, accionistas, empleados, proveedores, etc). En resumen, la 
reputación se basa no sólo en los resultados anteriores, sino también en la capacidad de una empresa para satisfacer las expectativas de los consumidores y para crear valor de futuro para sus grupos de interés (Wartick 2002). Algunos autores han sugerido que tomar pasos para construir una buena reputación puede ayudar a reforzar el éxito de una empresa que trabaja en la Red (Kotha, Rajgopal y Rindova 2001).

El conjunto de opiniones que se realizan en Internet, sobre una marca, bien o servicio, es lo que constituye la "reputación online" de una empresa. En principio, se trata de algo que queda fuera del alcance del empresario, ya que se supone que todas esas opiniones son vertidas por usuarios independientes. Pero no es del todo así, ya que la propia empresa puede intervenir en la creación de esta "reputación online" López 2010). Una reputación bien gestionada por parte de la empresa turística podría ser una herramienta estratégica de primer orden, en la medida que podría actuar como un elemento de fidelización de clientes, de mejora de su posicionamiento frente a los procesos de cooperación e integración, y finalmente, de atracción de recursos humanos cualificados (Rodríguez 2005)

\section{METODOLOGÍA}

Tras analizar las diferentes implicaciones de las redes sociales, el boca-oído electrónico, la Web 2.0 en el sector turístico y, las implicaciones que estos conceptos tienen para la creación de una reputación online, hemos considerado de interés centrarnos, por un lado, en la Web de viajes más importante del mundo, el caso de Tripadvisor y, por otro lado, la empresa de servicios española MiNube, por un concepto innovador dentro del sector, su rápida expansión, proyección internacional y, ante todo, por su carácter nacional (Miguéns, Baggio y Costa 2008; Ayeh, Au y Law 2013) .

Para el desarrollo de este trabajo nos hemos planteado la siguiente cuestión, ¿Cómo afectan los comentarios a la imagen de un destino o empresa turística? Para Jurca (2010) existe una clara dependencia entre los comentarios y puntuaciones dadas por diferentes clientes en un establecimiento hotelero. Las experiencias previas de otras personas, de este modo, generan expectativas respecto a la calidad del servicio que se extrae de los comentarios publicados en la diferentes Web.

Con la intención de extraer la información más destacable de cada comunidad virtual, se ha procedido a un esquema de división del trabajo que se planteará de igual manera para TripAdvisor y MiNube. En primer lugar, se ha realizado una ficha técnica a modo de comparativa, con los principales datos de ambas empresas. En segundo lugar, hemos querido estudiar cómo los usuarios, como generadores de contenidos, son los creadores de la información vertida en la Web. Y, por último, se hace referencia al e-WOM dentro de estas comunidades virtuales y los problemas y ventajas que acarrea, influyendo de esta manera en la imagen de marca.

\section{RESULTADOS}

Según Jain et al. (2012) en 2011 las opiniones de los viajeros crecieron un 69\% en TripAdvisor mientras que las agencias pertenecientes a las OTA's (Online Travel Agency) tan sólo aumentaron un 37\%, y la tendencia es que siga aumentando igual que aumenta el número de personas conectadas a redes sociales. No obstante, cabe destacar el estudio de O'Connor (2010) el cual realizó una muestra de hoteles de Londres para determinar qué causaba satisfacción e insatisfacción en los turistas alojados, donde destaca que son pocos los gerentes hoteleros o de restauración que gestionan su reputación a través de TripAdvisor, hecho que le 
planteó la cuestión de si los responsables de la industria turística se toman en serio los contenidos generados por los usuarios.

\subsection{TRIPADVISOR}

\subsubsection{Ficha técnica}

Hablar del desarrollo de Internet y el sector turístico requiere el nombrar a TripAdvisor como ejemplo de empresa que más ha crecido en el entorno 2.0 y que ha cambiado las reglas del juego para los participantes en la experiencia turística.

Con la intención de aglutinar gran parte de la información de esta Web se ha creado el siguiente cuadro: 


\section{TRIPADVISOR}

\begin{tabular}{|c|c|}
\hline Año de creación & 2.000 \\
\hline Lugar de creación & Estados Unidos \\
\hline \multirow{2}{*}{$\begin{array}{l}\text { Países en los que opera } \\
\text { Idiomas }\end{array}$} & 47 países \\
\hline & 28 idiomas \\
\hline Divisiones de la marca & $\begin{array}{l}\text { Más de } 20 \text { marcas de viajes: } \\
\text { www.airfarewatchdog.com,www.bokun.io,www.bookingbuddy.com,www.citymaps.com, www.cruisecritic.co } \\
\text { m, www.familyvacationcritic.com, www.flipkey.com, www.thefork.com (incluyendowww.lafourchette.com, w } \\
\text { ww.eltenedor.com, www.iens.nlwww.dimmi.com.au), www.gateguru.com, www.holidaylettings.co.uk, www.h } \\
\text { olidaywatchdog.com, www.housetrip.com, www.jetsetter.com, www.niumba.com, www.onetime.com, www.oy } \\
\text { ster.com, www.seatguru.com, www.smartertravel.com, www.tingo.com, www.vacationhomerentals.com y ww } \\
\text { w.viator.com. }\end{array}$ \\
\hline Funciones Web & $\begin{array}{l}\text { Índice de popularidad de los hoteles } \\
\text { Mapas } \\
\text { Vídeos y Fotografías subidos por los propios usuarios } \\
\text { Foros para viajeros } \\
\text { Guía de restaurantes y alojamientos } \\
\text { Búsqueda de vuelos y estimación de tarifas aéreas } \\
\text { Calculadora de alquileres vacacionales } \\
\text { Integrado con la red social Facebook (Friend of Friend) } \\
\text { Guía de ciudades para móviles (Mobile City Guides) } \\
\text { Alertas por email } \\
\text { Incrementar las funciones de la Web y seguir penetrando en nuevos mercados. }\end{array}$ \\
\hline \multirow{2}{*}{$\begin{array}{l}\text { Perspectiva de futuro } \\
\text { Aplicación móvil }\end{array}$} & Prepara su transformación en una red social de viajes \\
\hline & $\begin{array}{l}230 \text { millones de descargas } \\
\text { Más del } 50 \% \text { de los usuarios visita la Web a través de la App en tablet o móvil }\end{array}$ \\
\hline
\end{tabular}




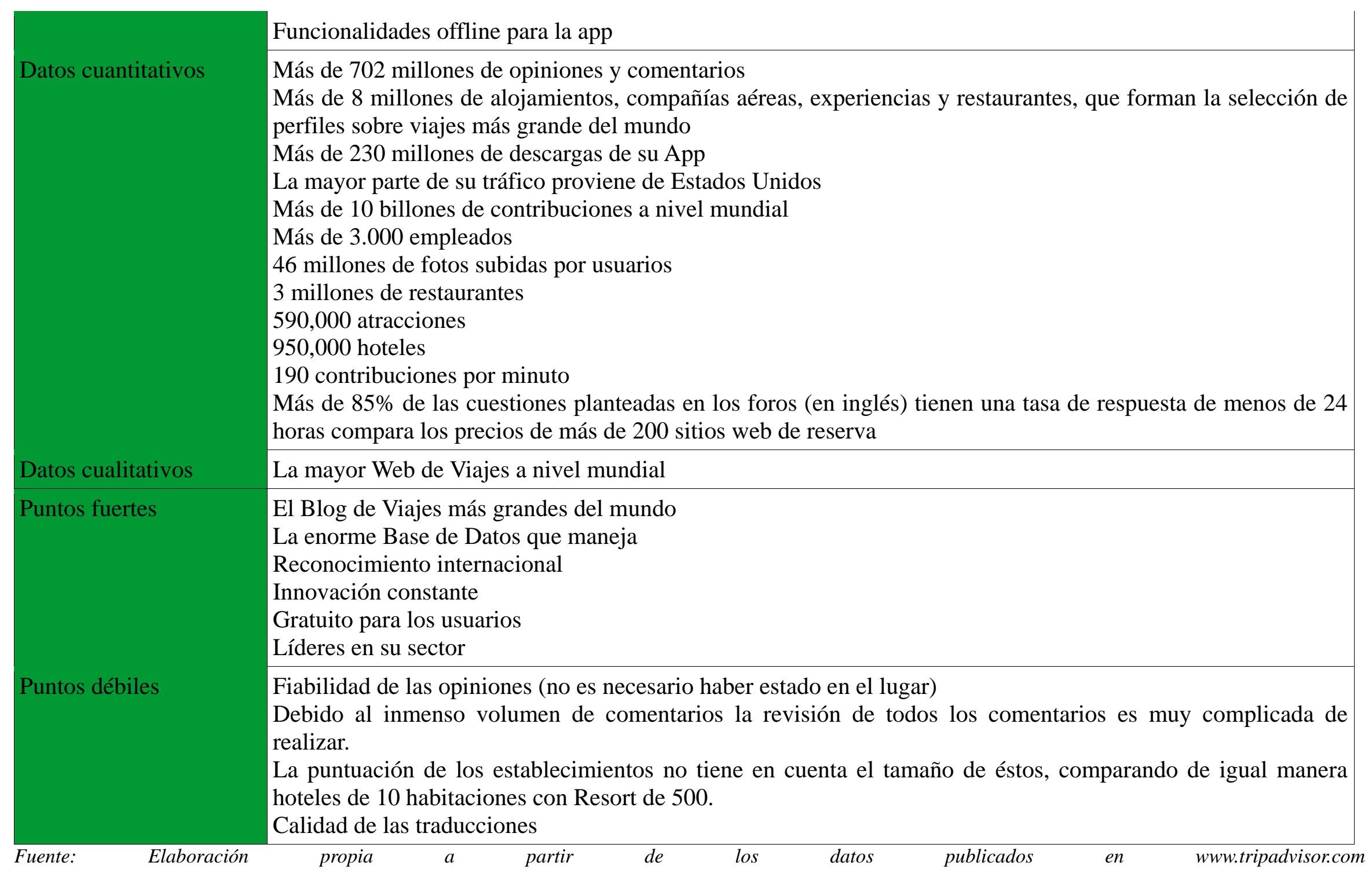




\subsection{2.- El papel de TripAdvisor como generador de contenido (UGC)}

El usuario en este tipo de Web es el que crea el contenido. Sin él no existiría la página, aunque, tal y como hemos mostrado en el cuadro-esquema, TripAdvisor tiene muchos otros servicios. Su punto fuerte es poder tener referencias de todos o casi todos los establecimientos turísticos existentes. TripAdvisor es el líder indiscutible en su sector, con una base de datos inmensa, motivo por el cual es muy interesante desde el punto de vista académico, para la extracción de datos tanto cualitativos como cualitativos de interés.

Cada establecimiento está dentro de un ranking creado por los propios clientes en función de diferentes parámetros como: limpieza, relación calidad-precio, trato del personal, ubicación, servicios que ofrece, etc.

En este sentido aporta una gran cantidad de información a los gerentes de los establecimientos, sabiendo de primera mano qué es lo que valora el cliente del local y cuáles son los aspectos que debería tener en cuenta para mejorar. Hasta hace poco, el empresario turístico podría obtener cierta información sobre el cliente y su experiencia a través de las llamadas encuestas de satisfacción, las cuales suponían un coste para el establecimiento y la mayoría de las veces no recibían el feedback correcto entre el cliente y la empresa. Ahora, con Webs como TripAdvisor se obtiene de manera gratuita información de gran valor para un producto intangible y tan cambiante.

Aunque también hay que destacar que si se hace una inadecuada gestión del establecimiento ésta se extenderá por toda la Red y quedará reflejado durante un tiempo en Internet, afectando a la reputación de la organización. Esta nueva realidad obliga a los hoteles a mejorar la calidad de sus servicios, no solo con el objetivo de fidelizar a los clientes, sino también para que los comentarios de éstos en Internet sean positivos, consiguiendo de esta manera que su reputación online se vea favorecida.

\subsection{3.- El e-WOM: inconvenientes $y$ ventajas. Influencia para crear una reputación online}

El boca-oído electrónico (e-WOM) juega un papel fundamental para la creación de una reputación online en el sector turístico. Teniendo por un lado, efectos positivos en la imagen de marca, cuando los comentarios son buenos pero, también puede tener efectos negativos sobre la reputación, cuando los comentarios vertidos por los usuarios de las comunidades virtuales no son nada buenos.

En la propia Web de la empresa definen a ésta como un lugar online que permite a los viajeros planificar sus viajes a través de informaciones fiables de otros viajeros. TripAdvisor, por tanto, considera que todos los comentarios publicados en su Web son reales y que no incurren en ningún fraude.

La aparición de fraudes y perfiles falsos son algunos de los principales problemas a los que se enfrentan muchos negocios presentes en Tripadvisor. En muchas ocasiones, han aparecido casos de perfiles que critican la calidad de un servicio de la competencia y elogian al máximo al suyo. Esta es una de las principales críticas que recibe esta empresa por parte de los empresarios, que consideran que muchos de los comentarios vertidos sobre ellos no son reales y que una sola persona podría arruinar la reputación de un hotel o destino. 
Para hacer un comentario en TripAdvisor no es necesario haber estado en el establecimiento, es suficiente con el registro de un perfil (que puede ser cierto o no) en la Web (al contrario que ocurre en página como Booking donde sólo pueden opinar clientes que hayan estado previamente alojados en el hotel). No obstante, TripAdvisor ofrece la opción, de contestar las opiniones de los clientes pudiendo aclarar lo sucedido. Además, si el empresario considera que muchas opiniones son de perfiles falsos y que dañan su imagen puede ponerse en contacto con la empresa y éstos determinarán si es posible la eliminación de ciertos comentarios.

En este sentido, el papel que pueden jugar portales como Tripadvisor, que publican millones de comentarios, ha originado numerosas disputas entre los hoteles y este tipo de Webs. Por eso, algunas páginas Web proponen filtros para acceder a la publicación de comentarios como es el pedir el localizador de la reserva para comprobar que realmente el cliente ha estado en el lugar del cual opina.

O'Connor (2010) en diversos estudios recientes confirma que los empresarios hoteleros no siguen ninguna estrategia para dirigir su reputación online en TripAdvisor. Hemos comprobado, tras analizar diversos comentarios en la Web, que son todavía gran parte el número de establecimientos hoteleros que no responden a las críticas vertidas en web como las nombradas, no dándoles la importancia necesaria. No obstante, según señala TripAdvisor, el $81 \%$ de las reseñas de los establecimientos son positivas, queriendo de esta manera no alarmar a la industria turística.

Así se desprende de un estudio realizado por PhoCusWright (2011) para TripAdvisor, que revela que el $74 \%$ de los que escriben opiniones lo hace para compartir una buena experiencia. Además, ver respuestas de la gerencia del hotel hace que se incrementen las posibilidades de reservar para un $57 \%$ de los encuestados.

En este nuevo modelo de negocio, las empresas deben diseñar estrategias de marketing dirigidas especialmente a las llamadas comunidades turísticas virtuales (Kim, Lehto y Morrison 2007; Rubio, Villaseñor y Yagüe 2017) . La planificación del viaje supone una parte importante de éste, donde se tendrán en cuenta muchos factores para conseguir el producto/servicio deseado. Los destinos y establecimientos turísticos deben tener personal cualificado en sus instalaciones que interactué con los clientes de la misma manera que ellos lo hacen. Tal y como señala (Jeacle y Carter 2011) los comentarios fraudulentos suponen en torno al $10 \%$ del contenido publicado en esta comunidad virtual, lo cual pone en riesgo la reputación de la empresa y/o destino.

Tripadvisor genera con su usuario un contrato de términos a la hora de participar en su Web, en la cual establece entre otros que la persona que participa con sus comentarios se compromete a no injuriar o lanzar falsos comentarios sobre una empresa, a la vez que fomenta la protección indicando la prohibición de hacer referencia a personas con nombres y apellidos o facilmente identificables (Gil, Barandalla y Idoeta 2017).

\subsection{MINUBE}

\subsubsection{Ficha Técnica}

El caso de MiNube es complicado de encontrar en la literatura, puesto que apenas hay menciones a día de hoy sobre su influencia en el sector turístico, al contrario de lo que nos ocurría con TripAdvisor que tal y como hemos venido comentando son muchos los estudios tratados sobre esta Web. MiNube menos conocida, creada por ingenieros españoles y que ante todo destaca por su proyección de futuro y su ritmo de crecimiento. 
Estamos ante un espacio de viajes sociales, donde el viajero comparte sus recomendaciones, fotografías, vídeos,... Una comunidad virtual que apuesta por hacer del viaje una experiencia inolvidable.

Hemos planteado el mismo cuadro resumen que para el caso de TripAdvisor. Para MiNube el volumen de negocio no tiene nada que ver con la Web líder en viajes, aun así podemos comprobar como un proyecto pequeño va aumentando día a día. A menudo y más concretamente en el sector turístico, nos fijamos sólo en las grandes empresas con inmensas cifras de negocio, dejando de un lado empresas como ésta que suponen una revolución en la experiencia turística. 


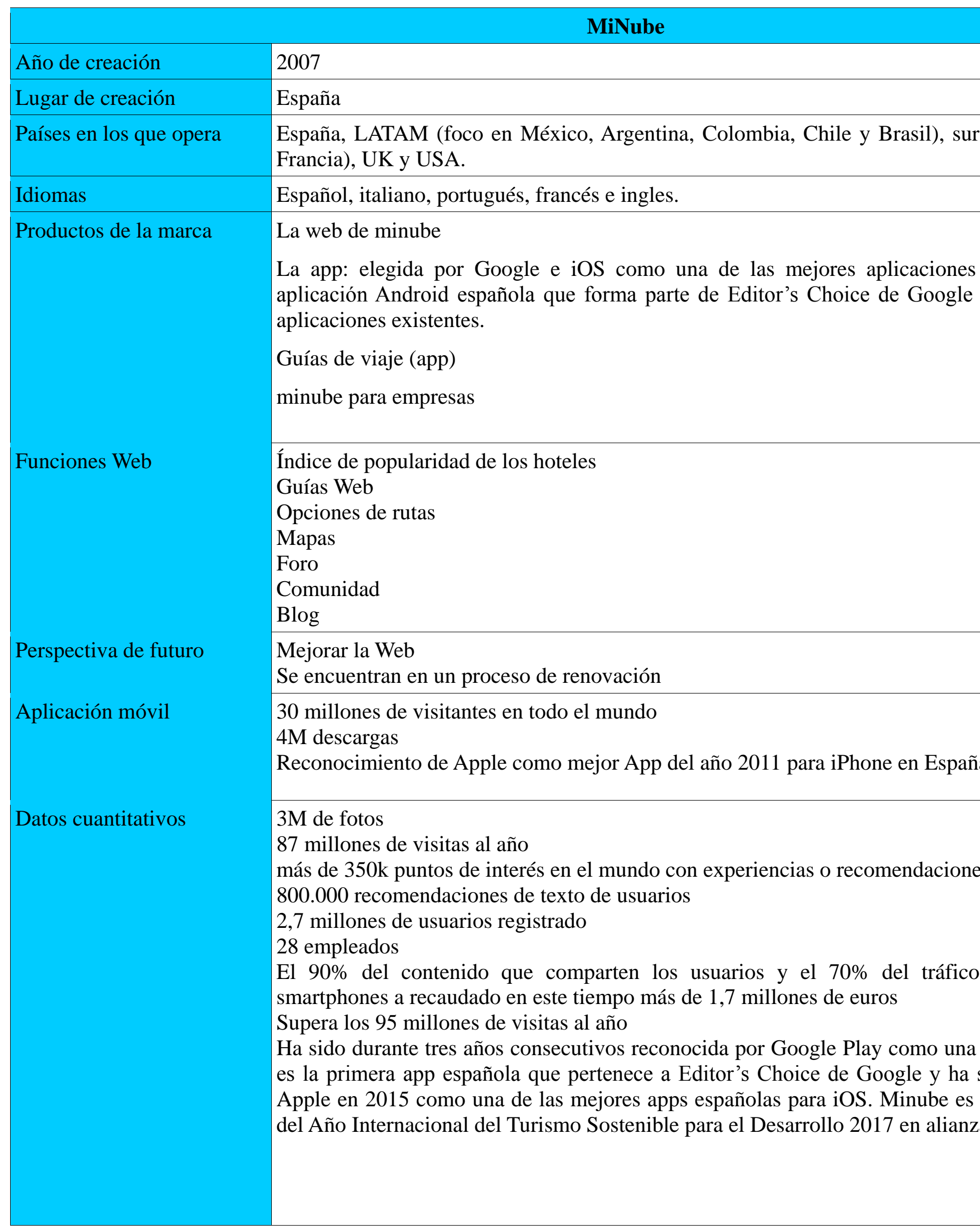




\begin{tabular}{|c|c|}
\hline Datos cualitativos & $\begin{array}{l}\text { Tres valores muy definidos: crecimiento sostenible, innovación y pa } \\
\text { comprometidos con el desarrollo de un turismo responsable y sostenible, la } \\
\text { colaborativo }\end{array}$ \\
\hline Puntos fuertes & $\begin{array}{l}\text { Creación de guías offline para el viajero } \\
\text { Quedada de viajeros } \\
\text { La aplicación se puede descargar sin conexión a internet } \\
\text { Elaboración de vídeos de promoción turística para empresas y } \\
\text { destinos. } \\
\text { Creación de un Observatorio Turístico donde publican informes sobre el } \\
\text { perfil del turista, los cambios en el sector, etc. } \\
\text { Colaboración con instituciones públicas turísticas para publicación } \\
\text { conjunta de guías y vídeos. } \\
\text { Sus contenidos se pueden compartir y comentar en Facebook y Twitter. } \\
\text { Los empleados de Minube revisan los comentarios hechos por usuarios } \\
\text { Colaboración con Rusticae y PlanB } \\
\text { Página Web y aplicación móvil totalmente intuitiva, atractiva y fácil de } \\
\text { usar. } \\
\text { Minube Schoolar }\end{array}$ \\
\hline Puntos débiles & $\begin{array}{l}\text { Todavía es una Red relativamente pequeña en comparación con TripAdvisor. } \\
\text { No ofrece la opción de réplica por parte del empresario } \\
\text { Cierto desconocimiento por parte del turista. } \\
\text { No ofrecen opiniones de alojamiento } \\
\text { Aunque apuestan sólo por comentarios positivos en la Web también hay } \\
\text { críticas sobre ciertos servicios. }\end{array}$ \\
\hline
\end{tabular}




\subsection{2.- El papel de MiNube como generador de contenido (UGC)}

Los usuarios de la comunidad virtual a la que hacemos referencia son los que con sus comentarios y aportaciones crean el contenido de la Web. Por ese motivo, son muchos los incentivos que desde la dirección de Minube se llevan a cabo para alentar al consumidor a relatar su experiencia. Para ello, se crean diferentes concursos, como el de seleccionar las diez mejores fotografías hechas por los usuarios, permitiendo que voten los usuarios de la Web y regalando al ganador una cámara fotográfica. A su vez, también se incentivan los encuentros de profesionales amateur o se premia a los usuarios que mayor valoración tengan por parte de otros miembros de la red a través de incentivos (programa de evangelizadores), consiguiendo de esta manera que los consumidores se trabajen un comentario original y genuino. Más de 96,5\% de los viajeros que comparten contenido en Minube no son evangelizadores, lo hacen de una forma natural porque simplemente les apasiona hablar de sus viajes. El $100 \%$ del contenido que se comparte en minube pasa tanto por un equipo humano como una una serie de sistemas que ayudan a priorizar dicha moderación.

Por lo tanto, y tal y como hemos mostrado, Minube tiene muy presente el concepto del usuario como generador del contenido, cuidando al detalle el trato al cliente, las opiniones vertidas, la personalización del servicio con el envío de una newsletter donde se reflejan las páginas que el usuario ha consultado, etc.

\subsection{3.- El e-WOM: inconvenientes $y$ ventajas. Influencia para crear una reputación online}

Aunque todavía no son muchas las empresas del sector turístico que tienen presentes las opiniones en esta red social, su importancia se revela a tener en cuenta en el futuro. El boca-oído influye de manera notable en la elección de un destino, sobre todo para aquellos viajeros que no saben dónde acudir y que, a través de las diferentes recomendaciones de otros usuarios se deciden por uno u otro destino.

Minube está basado principalmente en comentarios positivos, por lo cual, hasta el día de hoy, no ha supuesto ningún problema por parte de empresarios del sector. No obstante, como cada cliente puede tener una percepción de un lugar, a menudo encontramos ciertas críticas negativas dentro de comentarios positivos. El foco de Minube es conseguir que los viajeros compartan sus rincones favoritos en el mundo.

Minube es pionero en el uso de ciertas herramientas tecnológicas en España en el sector turístico. Se basan en el puro concepto 2.0, dándoles un enorme valor a los contenidos que los usuarios comparten en esta Web social. El control del contenido es vital, así pues, todo el contenido publicado por usuarios pasa por el control de una persona que se encarga de aceptar o no su publicación, cuidando de esta manera el contenido de la mejor manera.

Una de sus premisas es la de adaptar continuamente el contenido para hacerlo más visual, atractivo y usable. Minube se basa en un concepto de usabilidad, una Web muy sencilla y clara. Se basan en la innovación como un camino a seguir, creando nuevos servicios que ayuden al viajero casi semanalmente. Cabe destacar, que en el tema de los viajes el turista prefiere consultar en primer lugar a fuentes cercanas como familiares o amigos antes de acudir a canales oficiales de venta. 
En este caso, el concepto de reputación surge por las opiniones que sobre el destino en sí se experimenta, mientras que en el caso de TripAdvisor los comentarios se centran más en empresas que en destinos turísticos. En el caso de MiNube consiste en que los destinos (por ejemplo Portugal) pagan por agregar contenido de alta calidad para potenciarse como destino turístico en la comunidad de MiNube. Es parecido a los publirreportajes de las revistas.

El modelo de ingresos para el caso de MiNube se basa en tres partes: comisiones de hoteles, campañas de marketing para destinos turísticos y marcas relacionadas con viajes y display. Por su parte, TripAdvisor obtiene su fuente de ingresos principalmente a través de la publicidad.

\section{DISCUSIÓN Y CONCLUSIONES}

Los contenidos generados de manera gratuita a través de la participación del usuario adquieren un papel fundamental a la hora de adquirir un producto o servicio turístico. Así, el usuario valora más el contenido que no tiene un marcado carácter comercial. Es por ello que, se precisa de activos humanos conocedores del entorno 2.0 y de cómo entablar una conversación online, para que la empresa disponga de una imagen de marca de acuerdo con lo que quiere que los consumidores conozcan de ésta. Tal y como hemos analizado durante el desarrollo del presente trabajo, la Red ofrece grandes oportunidades para las empresas turísticas de conocer qué es lo que los consumidores demandan, sus debilidades y fortalezas como empresa y sus oportunidades de futuro. Siendo indispensable que toda empresa haga monitoreo de su reputación online, pero sobre todo esta actividad es de vital importancia para las empresas de la industria turística que se ven tan influenciada por las opiniones de millones de viajeros.

El propósito del presente trabajo ha consistido en determinar la potencialidad que la reputación online presenta para generar diferenciales de rentabilidad en el sector turístico español. Los datos observados a lo largo del desarrollo del trabajo, sobre la influencia de la reputación online en las empresas turísticas, confirman que aquellas empresas turísticas españolas que cuentan con un mayor stock de reputación online presentan también superiores niveles de rentabilidad. A su vez, autores como (Kotha, Rajgopal y Rindova 2001) desarrollaron la idea de que una buena reputación puede reforzar el éxito de una empresa que trabaja en la Red.

En diferentes estudios previos se analizó la importancia de TripAdvisor como generador de contenido, así como la credibilidad de los comentarios de los usuarios (Ayeh, Au y Law 2013). En otros estudios han analizado el papel de la dirección ante los comentarios en la Red (O'Connor 2010; Lee, Law y Murphy 2011) además de estudiar los ranking que se realizan en Tripadvisor y la reputación de los sitios Web (Vásquez 2011; Jeacle y Carter 2011)

Consideramos que trabajar la reputación online pasará a ser una necesidad para todas aquellas empresas que quieran participar del negocio turístico. De ahí el surgimiento de diferentes empresas que se encargan de gestionar la reputación online de los negocios (Reviewpro, Prestigiaonline, Gestión de la reputación online,...).

Finalmente, no se puede generar una reputación online o valor de marca positiva en la Red si no lo tenemos en el mundo offline. Se debe empezar creando un producto o servicio sólido y con un valor diferenciador, que aporte la tan ansiada "experiencia turística". En resumen, teniendo un producto turístico de calidad la gestión de la reputación online será mucho más sencilla. 
Antes eran las agencias de viajes (offline) las que recomendaban al cliente sobre empresas y destinos turísticos, mientras que ahora son los propios viajeros, los que viven la experiencia los que dan su opinión. ¿Desprofesionalización de los servicios? ¿Es necesario ser un experto en turismo para recomendar un lugar/empresa?

\section{LIMITACIONES Y FUTURAS LÍNEAS DE INVESTIGACIÓN}

En cuanto a las limitaciones del presente estudio se ha de partir de la base de que la investigación es una revisión de la literatura que incluye el análisis de dos empresas muy concretas en el campo de la reputación online, dejando fuera otras muchas empresas que influirán en la imagen de marca y que en este caso no han sido analizadas. Se ha analizado una pequeña parte del total de las comunidades turísticas virtuales. Los resultados, aunque basados en gran parte en la aportación de expertos en la materia, son difícilmente extrapolables a otros sectores.

La segunda limitación del presente trabajo se establece en lo cambiante del sector en cuanto al éxito de determinados portales Webs. Para Buhalis y Law (2008), la Web 2.0 en el caso de la industria turística representa una de las mayores innovaciones en materia de marketing online. El turista del siglo XXI es un turista experimentado, conocedor de muchos destinos y acostumbrado a alojarse en hoteles, usar diferentes medios de transporte, etc., un turista que necesita vivir una experiencia. Las herramientas online disponibles facilitan que el turista como ser social disponga de un lugar en que compartir, informar, comentar,... sus experiencias de viaje. Por lo tanto, páginas Web que hoy día son un éxito para la gestión de viajes puede que en un futuro no lo sean, debido a lo profundamente inestable e influenciable del sector objeto de estudio.

La evolución desde la primera Web, la conocida como Web 1.0, hasta el nacimiento de la Web semántica ha provocado un cambio en la forma en la que el sector turístico se comunica con el cliente. Los turistas han cambiado su comportamiento en la Red, pasando de ser meros espectadores de lo acontecido en la llamada Web 1.0 a creadores del contenido en la Web 2.0. En este contexto, el objetivo de este trabajo ha sido intentar analizar como el Turismo 2.0 ha cambiado la forma de comunicarse en el sector turístico, y de esta forma ha influido en la imagen de marca online, abriendo a su vez futuras líneas de investigación en el desarrollo de la Web semántica. La Web 3.0 supondrá el futuro de la planificación de los viajes online, sistemas inteligentes que facilitarán la búsqueda de información. Hemos considerado de interés el no quedarnos sólo al amparo de la Web 2.0 y su influencia para la comunicación turística, sino que hemos querido plantear como la reputación online de las empresas turística será fundamental para la selección de un destino, puesto que el desarrollo de la Web semántica aglutinará todo lo comentado en la Web sobre una empresa y su importancia será crucial para el éxito de las organizaciones del sector.

Las investigaciones futuras podrían abarcar la importancia de la reputación online en un entorno 3.0, puesto que hasta la fecha ambos conceptos de reputación y Web semántica apenas han sido tratados en la literatura científica. Se trata de saber, desde el punto de vista empresarial, cómo llegar al cliente en un entorno futuro.

Esta área de estudio precisa de una investigación interdisciplinar con las bases de otros campos científicos como la etnografía, la sociología, la psicología, la informática, etc. Metodológicamente hablando, parece que hay una escasez de estudios de campo donde se usen más de una comunidad virtual (como es el caso de TripAdvisor) y la casi total inexistencia del empleo de métodos cualitativos para el análisis de los comentarios en las principales Web. Consideramos que la investigación en el sector turístico, tanto a nivel 
internacional como en el caso español, y por su importancia para el tejido económico, necesita de más originalidad en los temas abordados y en los métodos de investigación utilizados. Después de lo estudiado, parece que la investigación científica va unos pasos por detrás del desarrollo tecnológico y de las publicaciones no académicas.

Aunque las empresas turísticas son consideradas las primeras en adoptar las nuevas tecnologías, la investigación muestra que existe una infrautilización y abandono en la explotación de estas tecnologías. Por otra parte, debido a las inconsistencias en la literatura, que examinan el papel de papel de la Web en la creación de una reputación turística online se convierte en un tema de investigación emergente.

Una de las principales dificultades del estudio proviene del hecho de que TripAdvisor no proporciona información sobre su empresa más allá de la publicada en su Web, lo cual limita bastante la investigación al respecto, debiendo basarnos en una información completamente sesgada y con una clara intención comercial.

A su vez, los investigadores deberían estudiar la formación de expectativas de los diferentes grupos de turistas según la nacionalidad, la participación o la familiaridad con el destino. Esto es debido a que el papel en la formación de expectativas puede variar de un turista a otro. Por otra parte, futuros trabajos deberían hacer hincapié en el papel de las motivaciones en el proceso de formación de expectativas. Las expectativas están estrechamente relacionadas con la satisfacción y ello repercutirá en los comentarios online. Así pues, crear unas expectativas como destino o empresa demasiado altas supondrán también un problema a la hora de comparar la imagen real con la creada.

En este trabajo se han analizado dos comunidades virtuales pero esto no supone que se desarrollen más en los próximos años e incluso meses y que se hagan con gran parte del mercado. Nosotros consideramos que el uso de técnicas de análisis cualitativo para el estudio de las páginas Web de viajes podría enriquecer el estudio llevado a cabo. Este problema del trabajo realizado será tratado en líneas de investigación futuras.

En resumen, a raíz de esta investigación se nos plantean diversos interrogantes como ¿puede una empresa posicionarse entre los primeros puestos sin comentarios de clientes reales? ¿Debería existir un control de los comentarios publicados en las Webs de viajes? ¿Se puede tener reputación offline y no tener reputación online, y viceversa?...

Finalmente, el uso de otras redes sociales para la gestión de los viajes como Facebook o Twitter, con la gran cantidad de seguidores y su influencia en la gestión de la imagen de marca, también sería interesante a tener en cuenta para futuras investigaciones en esta área.

\section{BIBLIOGRAFÍA}

ARMSTRONG, A. y HAGEL, J., 2000. Chapter 6 - The Real Value of Online Communities**Reprinted with permission from Harvard Business Review (May-June 1996): 134-141. Copyright (C) 1996 by the President and Fellows of Harvard College; all rights reserved. En: E.L. LESSER, M.A. FONTAINE y J.A. SLUSHER (eds.), Knowledge and Communities [en línea]. Boston: Butterworth-Heinemann, pp. 85-95. [Consulta: 4 diciembre 2018]. ISBN 978-0-7506-7293-1. Disponible http://www.sciencedirect.com/science/article/pii/B9780750672931500093.

ARSAL, I., WOOSNAM, K.M., BALDWIN, E.D. y BACKMAN, S.J., 2010. Residents as Travel Destination Information Providers: An Online Community Perspective. Journal of 
Travel Research, vol. 49, no. 4, pp. 400-413. ISSN 0047-2875. DOI 10.1177/0047287509346856.

AYEH, J.K., AU, N. y LAW, R., 2013. "Do We Believe in TripAdvisor?" Examining Credibility Perceptions and Online Travelers' Attitude toward Using User-Generated Content. Journal of Travel Research, vol. 52, no. 4, pp. 437-452. ISSN 0047-2875. DOI 10.1177/0047287512475217.

BECHWATI, N.N. y NASR, N.I., 2011. Understanding Triggers of Offline and Online Consumer Recommendations. Journal of Consumer Satisfaction, Dissatisfaction and Complaining Behavior; Provo, vol. 24, pp. 56-68. ISSN 08998620.

BENÍTEZ, V.A., LÓPEZ, M.T. y GUTIÉRREZ, I.M., 2018. Turista 2.0, comportamiento y uso de los medios sociales. Chasqui. Revista Latinoamericana de Comunicación, vol. 0, no. 137, pp. 209-225. ISSN 1390-924X. DOI 10.16921/chasqui.v0i137.3513.

BITNER, M.J., BOOMS, B.H. y TETREAULT, M.S., 1990. The Service Encounter: Diagnosing Favorable and Unfavorable Incidents. Journal of Marketing, vol. 54, no. 1, pp. 71-84. ISSN 0022-2429. DOI 10.2307/1252174.

BUHALIS, D. y LAW, R., 2008. Progress in information technology and tourism management: 20 years on and 10 years after the Internet-The state of eTourism research. Tourism Management, vol. 29, no. 4, pp. 609-623. ISSN 0261-5177. DOI 10.1016/j.tourman.2008.01.005.

BURGESS, L., PARISH, B. y ALCOCK, C., 2011. To what extent are regional tourism organisations (RTOs) in Australia leveraging the benefits of web technology for destination marketing and eCommerce? Electronic Commerce Research, vol. 11, no. 3, pp. 341-355. ISSN 1572-9362. DOI 10.1007/s10660-011-9077-1.

CHUN, R. y DAVIES, G., 2001. E-reputation: The role of mission and vision statements in positioning strategy. Journal of Brand Management, vol. 8, no. 4, pp. 315-333. ISSN 14791803. DOI 10.1057/palgrave.bm.2540031.

CONSOLI, R. y BAGLIERI, D., 2009. Collaborative innovation in tourism: managing virtual communities. The TQM Journal, vol. 21, no. 4, pp. 353-364. ISSN 1754-2731. DOI 10.1108/17542730910965065.

DI PIETRO, L. y PANTANO, E., 2012. An Empirical Investigation of Social Network Influence on Consumer Purchasing Decision: The Case of Facebook. [en línea]. SSRN Scholarly Paper. Rochester, NY: Social Science Research Network. [Consulta: 4 diciembre 2018]. ID 1978423. Disponible en: https://papers.ssrn.com/abstract=1978423.

FOMBRUN, C.J., 2017. Corporate Reputations as Economic Assets. The Blackwell Handbook of Strategic Management [en línea]. S.1.: John Wiley \& Sons, Ltd, pp. 285-308. [Consulta: 4 diciembre 2018]. ISBN 978-1-4051-6402-3. Disponible en: https://onlinelibrary.wiley.com/doi/abs/10.1111/b.9780631218616.2006.00011.x.

GIL, Á.R., BARANDALLA, I.J. y IDOETA, C.M., 2017. Reputación corporativa online en la hotelería: el caso TripAdvisor. Esic market, no. 158, pp. 579-608. ISSN 0212-1867.

GOMIS, J.M.F., 2008. LOS BLOGS DENTRO DEL TURISMO 2.0. ROTUR: Revista de Ocio y Turismo, vol. 1, no. 1, pp. 135-152. ISSN 1888-6884. DOI 10.17979/rotur.2008.1.1.1230. 
GRETZEL, U., 2006. Consumer generated content - trends and implications for branding. $e$ Review of Tourism Research, vol. 4, no. 3, pp. 9-11. ISSN 1941-5842.

HENNIG-THURAU, T., GROTH, M., PAUL, M. y GREMLER, D.D., 2006. Are All Smiles Created Equal? How Emotional Contagion and Emotional Labor Affect Service Relationships. Journal of Marketing, vol. 70, no. 3, pp. 58-73. ISSN 15477185(Electronic),0022-2429(Print). DOI 10.1509/jmkg.70.3.58.

HERBIG, P., MILEWICZ, J. y GOLDEN, J., 1994. A model of reputation building and destruction. Journal of Business Research, vol. 31, no. 1, pp. 23-31. ISSN 18737978(Electronic),0148-2963(Print). DOI 10.1016/0148-2963(94)90042-6.

HERNÁNDEZ-MÉNDEZ, J., MUÑOZ-LEIVA, F. y SÁNCHEZ-FERNÁNDEZ, J., 2015. The influence of e-word-of-mouth on travel decision-making: consumer profiles. Current Issues in Tourism, vol. 18, no. 11, pp. 1001-1021. ISSN 1368-3500. DOI 10.1080/13683500.2013.802764.

JAIN, M., KHALIL, S., LE, A.N.-H. y CHENG, J.M.-S., 2012. The glocalisation of channels of distribution: a case study. [en línea]. [Consulta: 13 diciembre 2018]. Disponible en: https://www.ingentaconnect.com/content/mcb/001/2012/00000050/00000003/art00009.

JANSSON, A., 2018. Rethinking post-tourism in the age of social media. Annals of Tourism Research, vol. 69, pp. 101-110. ISSN 0160-7383. DOI 10.1016/j.annals.2018.01.005.

JARVENPAA, S.L., TRACTINSKY, N. y SAARINEN, L., 1999. Consumer Trust in an Internet Store: a Cross-Cultural Validation. Journal of Computer-Mediated Communication [en línea], vol. 5, no. 2. [Consulta: 13 diciembre 2018]. DOI 10.1111/j.10836101.1999.tb00337.x. Disponible https://academic.oup.com/jcmc/article/5/2/JCMC526/4584190.

JEACLE, I. y CARTER, C., 2011. In TripAdvisor we trust: Rankings, calculative regimes and abstract systems. Accounting, Organizations and Society, vol. 36, no. 4, pp. 293-309. ISSN 0361-3682. DOI 10.1016/j.aos.2011.04.002.

JØSANG, A., ISMAIL, R. y BOYD, C., 2007. A Survey of Trust and Reputation Systems for Online Service Provision. Decis. Support Syst., vol. 43, no. 2, pp. 618-644. ISSN 0167-9236. DOI 10.1016/j.dss.2005.05.019.

JURCA, M.A., 2010. The Forms Of Unconventional Advertising - A Theoretical Approach. Management and Marketing Journal, vol. VIII, no. 2, pp. 323-333.

KIM, D.-Y., LEHTO, X.Y. y MORRISON, A.M., 2007. Gender differences in online travel information search: Implications for marketing communications on the internet. Tourism Management, vol. 28, no. 2, pp. 423-433. ISSN 0261-5177. DOI 10.1016/j.tourman.2006.04.001.

KOTHA, S., RAJGOPAL, S. y RINDOVA, V., 2001. Reputation Building and Performance: An Empirical Analysis of the Top-50 Pure Internet Firms. European Management Journal, vol. 19 , no. 6, pp. 571-586.

KUSUMASONDJAJA, S., SHANKA, T. y MARCHEGIANI, C., 2012. Credibility of online reviews and initial trust: The roles of reviewer's identity and review valence. Journal of Vacation Marketing, vol. 18, no. 3, pp. 185-195. ISSN 1356-7667. DOI $10.1177 / 1356766712449365$. 
LEE, H. "Andy", LAW, R. y MURPHY, J., 2011. Helpful Reviewers in TripAdvisor, an Online Travel Community. Journal of Travel \& Tourism Marketing, vol. 28, no. 7, pp. 675688. ISSN 1054-8408. DOI 10.1080/10548408.2011.611739.

LEE, K.-H. y HYUN, S.S., 2018. The effects of tourists' knowledge-sharing motivation on online tourist community loyalty: the moderating role of ambient stimuli. Current Issues in Tourism, vol. 21, no. 13, pp. 1521-1546. ISSN 1368-3500. DOI 10.1080/13683500.2016.1145197.

LUQUE-MARTÍNEZ， T., CASTAÑEDA-GARCÍA， J.A., FRÍAS-JAMILENA， D.M., MUÑOZ-LEIVA, F. y RODRÍGUEZ-MOLINA, M.A., 2007. Determinants of the Use of the Internet as a Tourist Information Source. The Service Industries Journal, vol. 27, no. 7, pp. 881-891. ISSN 0264-2069. DOI 10.1080/02642060701570586.

MANGOLD, W.G. y FAULDS, D.J., 2009. Social media: The new hybrid element of the promotion mix. Business Horizons, vol. 4, no. 52, pp. 357-365. ISSN 0007-6813. DOI 10.1016/j.bushor.2009.03.002.

MIGUÉNS, J., BAGGIO, R. y COSTA, C., 2008. Social media and Tourism Destination: TripAdvisor Case Study. IASK Advances in Tourism Research 2008 (ATR2008) [en línea]. S.l.: IASK, pp. 194-199. [Consulta: 18 diciembre 2018]. Disponible en: https://iris.unibocconi.it/handle/11565/2459791\#.XBjDmBSmk8o.

MORO, S. y RITA, P., 2018. Brand strategies in social media in hospitality and tourism. International Journal of Contemporary Hospitality Management, vol. 30, no. 1, pp. 343-364. ISSN 0959-6119. DOI https://doi.org/10.1108/IJCHM-07-2016-0340.

O'CONNOR, P., 2010. Managing a Hotel's Image on TripAdvisor. Journal of Hospitality Marketing \& Management, vol. 19, no. 7, pp. 754-772. ISSN 1936-8623. DOI 10.1080/19368623.2010.508007.

PAN, B., MACLAURIN, T. y CROTTS, J.C., 2007. Travel Blogs and the Implications for Destination Marketing. Journal of Travel Research, vol. 46, no. 1, pp. 35-45. ISSN 00472875. DOI 10.1177/0047287507302378.

PARAMESWARAN, M. y WHINSTON, A.B., 2007. Social Computing: An Overview. Communications of the Association for Information Systems [en línea], vol. 19, no. 1. ISSN 1529-3181. DOI 10.17705/1CAIS.01937. Disponible en: https://aisel.aisnet.org/cais/vol19/iss1/37.

RICCI, F., ROKACH, L. y SHAPIRA, B., 2015. Recommender Systems: Introduction and Challenges. En: F. RICCI, L. ROKACH y B. SHAPIRA (eds.), Recommender Systems Handbook [en línea]. Boston, MA: Springer US, pp. 1-34. [Consulta: 4 diciembre 2018]. ISBN 978-1-4899-7637-6. Disponible en: https://doi.org/10.1007/978-1-4899-7637-6_1.

RODRÍGUEZ, F.J.G., 2005. DIFERENCIAS REGIONALES Y ESTRUCTURALES DE REPUTACIÓN EN LA EMPRESA HOTELERA ESPAÑOLA. Cuadernos de Turismo, no. 15, pp. 135-148. ISSN 1989-4635.

RUBIO, N., VILLASEÑOR, N. y YAGÜE, M.J., 2017. Modelo de experiencia en la comunidad virtual desde la perspectiva de la cocreación [en línea]. S.1.: ESIC. [Consulta: 13 diciembre 2018]. ISBN 978-84-17129-34-7. Disponible en: https://idus.us.es/xmlui/handle/11441/78103.

RUIZ-MAFE, C., BIGNE-ALCAÑIZ, E., SANZ-BLAS, S. y TRONCH, J., 2018. Does social climate influence positive eWOM? A study of heavy-users of online communities. $B R Q$ 
Business Research Quarterly, vol. 21, no. 1, pp. 26-38. ISSN 2340-9436. DOI 10.1016/j.brq.2017.12.001.

SMITH, D., MENON, S. y SIVAKUMAR, K., 2005. Online peer and editorial recommendations, trust, and choice in virtual markets. Journal of Interactive Marketing, vol. 19, no. 3, pp. 15-37. ISSN 1520-6653. DOI 10.1002/dir.20041.

SOLIS, B. y BREAKENRIDGE, D.K., 2009. Putting the Public Back in Public Relations: How Social Media Is Reinventing the Aging Business of PR. S.1.: FT Press. ISBN 978-0-13701126-1.

STANDING, C., TANG-TAYE, Jean-Pierre y BOYER, M., 2014. The Impact of the Internet in Travel and Tourism: A Research Review 2001-2010. Journal of Travel \& Tourism Marketing, vol. 31, pp. 82-113. DOI 10.1080/10548408.2014.861724.

TRUSOV, M., BODAPATI, A.V. y BUCKLIN, R.E., 2010. Determining Influential Users in Internet Social Networks. Journal of Marketing Research, vol. 47, no. 4, pp. 643-658. ISSN 0022-2437. DOI 10.1509/jmkr.47.4.643.

TUSSYADIAH, I.P. y FESENMAIER, D.R., 2009. Mediating Tourist Experiences: Access to Places via Shared Videos. Annals of Tourism Research, vol. 36, no. 1, pp. 24-40. ISSN 01607383. DOI 10.1016/j.annals.2008.10.001.

VAN DOLEN, W., LEMMINK, J., DE RUYTER, K. y DE JONG, A., 2002. Customer-sales employee encounters: a dyadic perspective. Journal of Retailing, vol. 78, no. 4, pp. 265-279. ISSN 0022-4359. DOI 10.1016/S0022-4359(02)00067-2.

VÁSQUEZ, C., 2011. Complaints online: The case of TripAdvisor. Journal of Pragmatics, vol. 43, no. 6, pp. 1707-1717. ISSN 0378-2166. DOI 10.1016/j.pragma.2010.11.007.

VICKERY, G. y WUNSCH-VINCENT, S., 2007. Participative Web And User-Created Content: Web 2.0 Wikis and Social Networking. Paris, France, France: Organization for Economic Cooperation and Development (OECD). ISBN 978-92-64-03746-5.

WARTICK, S.L., 2002. Measuring Corporate Reputation: Definition and Data. Business \& Society, vol. 41, no. 4, pp. 371-392. ISSN 0007-6503. DOI 10.1177/0007650302238774.

WEIGELT, K. y CAMERER, C., 1988. Reputation and corporate strategy: A review of recent theory and applications. Strategic Management Journal, vol. 9, no. 5, pp. 443-454. ISSN 1097-0266. DOI 10.1002/smj.4250090505.

WESTBROOK, R.A., 1987. Product/Consumption-Based Affective Responses and Postpurchase Processes. JMR, Journal of Marketing Research; Chicago, vol. 24, no. 3, pp. 258. ISSN 00222437.

YACOUEL, N. y FLEISCHER, A., 2012. The Role of Cybermediaries in Reputation Building and Price Premiums in the Online Hotel Market. Journal of Travel Research, vol. 51, no. 2, pp. 219-226. ISSN 0047-2875. DOI 10.1177/0047287511400611.

YOO, K.-H. y GRETZEL, U., 2009. Comparison of Deceptive and Truthful Travel Reviews. En: W. HÖPKEN, U. GRETZEL y R. LAW (eds.), Information and Communication Technologies in Tourism 2009. S.1.: Springer Vienna, pp. 37-47. ISBN 978-3-211-93971-0 